\title{
Interventions With Family Caregivers
}

Jonathan G. Sandberg

Brigham Young University - Provo, jonathan_sandberg@byu.edu

Follow this and additional works at: https://scholarsarchive.byu.edu/facpub

Part of the Other Social and Behavioral Sciences Commons

\section{Original Publication Citation}

Sandberg, J.G. (2006). Interventions with family caregivers. Handbook and Families and Health. (Eds.) Crane, R., \& Marshall, Sage: Thousand Oaks, CA.

\section{BYU ScholarsArchive Citation}

Sandberg, Jonathan G., "Interventions With Family Caregivers" (2006). Faculty Publications. 4071. https://scholarsarchive. byu.edu/facpub/4071

This Book Chapter is brought to you for free and open access by BYU ScholarsArchive. It has been accepted for inclusion in Faculty Publications by an authorized administrator of BYU ScholarsArchive. For more information, please contact ellen_amatangelo@byu.edu. 


\title{
снартев 24
}

\section{Interventions With Family Caregivers}

\author{
Jonathan G. SANDBERG
}

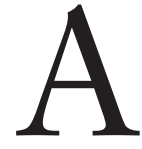

s a typical self-centered teenager, I struggled to find anything good about grandma moving in with us. Grandma required a lot of help with stairs, dressing, bathing; I noticed this brought a new kind of stress into our home. I think it was particularly hard on my mother, who provided most of the daily care for her mother-in-law. Living in a basement bedroom, I could escape from the stress if I wanted. I did not share a bathroom or hallway, I did not have to listen to incoherent talk and cries in the night as Alzheimer's disease began to take its toll. Yet, I knew that my parents and some siblings slept less, worried more, and lost some freedom.

Over time, I began to feel drawn to grandma. Though I was still angry on occasion, I also found tender moments with her. I appreciated more the few small opportunities I had to help grandma or my parents as they were helping her. I remember sitting side by side with her one Christmas Eve long after she had lost the ability to carry on a conversation-at least I thought. As a favorite hymn began to play she nudged me and said, "We better stand as we sing this one." I helped her to her feet and she proceeded to sing all three verses from memory as we stood arm in arm. At that moment I could see her; she was still in there somewhere, that deeply spiritual, strong, and committed music teacher. It was a memorable moment to connect with her on that level. I am particularly grateful for that shared experience because shortly thereafter my family made the difficult decision to move grandma to a long-term care facility. She then passed away while I was far from home.

As I reflect upon this experience, I am reminded of several ironies regarding family caregiving. The emotional and physical work of caregiving can be so burdensome, yet somehow that service and sacrifice can bind hearts together in a powerful way. Caregiving is a family affair, yet individual members can feel isolated in their own experience, even lonely when surrounded by others. Perhaps caregiving is best described as a great tension of opposites: burden and privilege, isolation and connection, pain and joy. In some ways it also represents the past, present, and future, with all its fears and dreams, rolled up in one. However described, caregiving in a family setting is a commonly experienced and well-researched phenomenon. 


\section{FAMILY CAREGIVING: PREVALENCE AND TRENDS}

A number of large-scale demographic trends have greatly influenced the number of families providing daily care for an aging loved one. First, people across the world are living longer, much longer (Armas, 2003). For example, in the United States the average life expectancy has nearly doubled, from the mid-forties in 1900 to nearly 80 in the year 2000 (Corcoran, Fairchild-Kienlen, \& Phillips, 2000). Because of combined trends of overall decreases in birth rates and the aging of the baby boom generation, the largest segment of the U.S. population is quickly approaching age 65 . By 2030, it is estimated that the number of older adults (65 and older) will jump to 70 million, a full $20 \%$ of the overall population and twice the number in 1999 (Administration on Aging [AoA], 2000). The fastest growth during that time is projected to occur among minority older adults, a $219 \%$ growth rate as compared to only an $81 \%$ increase for the white population (AoA, 2000).

The dramatic increase in life expectancy can be attributed to many factors, perhaps chief among them the tremendous ability of modern medicine to preserve and prolong life. For this reason, many older adults are living longer with chronic and debilitating physical and mental conditions (Zarit \& Zarit, 1998). These trends, prolonged life and fewer births, combine to present a staggering challenge to family caregiving. At the same time a greater number of older adults may need daily care, there are fewer family members to provide it. Research near the turn of the 21st century suggested that nearly one-quarter of all homes in the U.S. already had at least one caregiver (Corcoran et al., 2000). At that time, one national survey concluded that a typical caregiver was a married woman in her 40s, working full time and providing care for an older care recipient (average age 77 ). The average caregiver provided 18 hours of care a week, with one in five providing more than 40 hours (Corcoran et al., 2000). Clearly, these trends will only increase as the population ages. Since families are always "the first line of defense ... [for providing] extensive care, often at considerable sacrifice of their own health and well-being," it is appropriate that researchers, policy makers, and clinicians pay particular attention to the issues of family caregiving (Zarit \& Zarit, 1998, p. 290).

\section{THE EXPERIENCE OF PROVIDING CARE}

In the last two decades of the 20th century, over 400 empirical studies solely on the psychological effects of caregiving were published (Pinquart \& Sorensen, 2003a). These papers, along with published case studies and narratives, paint caregiving as both a challenging and a rewarding task. Caregiver burden is the most researched topic among this literature. Major theorists have noted that caregivers experience stress or burden on two levels. Primary stressors are related to direct care of the recipient and the behavioral, emotional, and physical tasks required in that process. Secondary stressors are related to the impact of caregiving on other aspects of the caregiver's life, such as marriage, employment, and personal finances (Zarit \& Zarit, 1998).

A meta-analysis of caregiver burden research has clearly shown that caregivers experience lower levels of physical health and subjective well-being and greater levels of stress and depression than non-caregivers (Pinquart \& Sorensen, 2003b). Other studies have noted that family caregivers spend less time interacting with others in their social network, particularly outside the home, and experience increased conflict with family members regarding caregiving responsibilities (Pillemer, Suitor, \& Wethington, 2003). Study after study has shown that caregivers 
are vulnerable to multiple physical and mental health problems and are frequently isolated in their suffering.

Researchers have also sought to identify which aspects of caregiving prove most troublesome for caregiving. Theorists have hypothesized that care recipients' impairment (cognitive, physical, or behavior problems), caregiver involvement (amount of care, duration), and perception of uplift all play a major role in determining caregiver burden outcomes (Pinquart \& Sorensen, 2003a). A second meta-analysis suggested that behavior problems of the care recipient are most strongly associated with caregiver burden and depression, although levels of physical and cognitive impairment are also associated with burden and depression at less significant levels (Pinquart \& Sorensen, 2003a). Clearly, interventions that can serve to decrease burden, particularly the strain of care recipient behavior problems, and help caregivers stay socially engaged are of great worth to both families and society at large.

It is also important to note that many caregivers experience satisfaction and joy as a result of their service (Kramer, 1997). Some studies report that up to $75 \%$ of caregivers report that providing care helps them feel useful (i.e., facilitates self worth, confidence) (Myers, 2003). Other studies suggest that caregiving fosters an increased closeness to the care recipient and a sense of fulfilling one's duty (Pinquart \& Sorensen, 2003b). When caregivers are able to experience a sense of mastery in caregiving tasks, they can also experience a sense of accomplishment and control that may serve to offset burden (Kramer, 1997; Wilken, Altergott, \& Sandberg, 1996). A quotation from one caregiver highlights how the sweet can accompany the bitter while providing care: "This year and a half [of caregiving] has been so hard, but I can honestly say my mother and I have had some of the most tender times of our lives" (Hargrave, 2002, p. 26).

\section{THE EXPERIENCE OF RECEIVING CARE}

As broad and almost overwhelming as the caregiving literature has become, there is a similarly striking absence of research on the experience of care recipients. In the majority of caregiving studies, the receiver of care is viewed objectively in terms of her or his impairment and behavior and its impact on caregiver burden (Lyons, Zarit, Syer, \& Whitlatch, 2002). Even in cases where the recipient's cognitive functioning is good and her or his input is sought, few studies go beyond assessing mental health status or decisions around institutionalization (Lyons et al., 2002). Non-caregiving research related to normative physical decline and loss (e.g., retirement, change of residence) associated with healthy aging suggests that decreased autonomy and independence can be very difficult for older adults (Sandberg \& Platt, 2001). Such decline and loss can make the care recipient more vulnerable to social withdrawal and inactivity as well as depression (Hargrave \& Hanna, 1997; Pillemer et al., 2003).

One group of researchers integrated both caregiver and care recipient perspectives and noted givers and receivers differed little over care recipient needs, but disagreed significantly on their appraisal of caregiving difficulties (Lyons et al., 2002). Specifically, the authors noted that caregivers perceived less support and cooperation than care recipients and that this discrepancy increased as the level of caregiving difficulties increased as well. Interestingly, the authors also point out that the caregiver's appraisal of relationship strain predicted the level of discrepancy and level of caregiving difficulties, indicating that the quality and nature of the relationship between giver and recipient has an impact on caregiver experience.

A second study (Conger \& Marshall, 1998) that looked at both caregivers and care 
recipients provides greater insight into the experience of those who receive care. Drawing upon data from a series of longitudinal qualitative interviews with caregiving dyads, the authors theorize that care recipients may enter into a redefinition period where they shift long-held views and beliefs about self and relationships. Specifically, Conger and Marshall (1998) suggested that illness often brings a change in roles and an accompanying sense of loss for care recipients. The process of redefining self is one of acknowledging change and coming to terms with new priorities and less control and autonomy in one's life. Where cognitively possible, the care recipient may also seek to redefine his or her marital relationship as the couple creates shared meaning around the multitude of changes in their lives. In cases where the process of redefining life is beyond the reach of care recipients or their caregivers, the stresses and challenges of daily caregiving may soon take their toll on the relationship.

\section{CULTURAL AND \\ GENDER DIFFERENCES IN} THE CAREGIVING EXPERIENCE

As mentioned, the first half of the 21st century will likely be known as an age of "graying," particularly among culturally and racially diverse groups. While the nonHispanic white elderly population will likely double by the year 2050, the African American elderly population will quadruple, the Latino elderly population will increase by 7 times, the Asian/Pacific Islander by 6.5, and the American Indian population will increase to 3.5 times its current size (DilworthAnderson, Williams, \& Gibson, 2002). With these trends in mind, there is a clear social imperative to increase understanding regarding the needs of dependent older adults in racially and ethnically diverse groups (Dilworth-Anderson et al., 2002). Because cultural values, norms, and perceptions influence all aspects of caregiving, from an understanding of how disease is contracted to what "good" caregivers should and should not do (Dilworth-Anderson \& Gibson, 2002), it is important that helping professionals do not fall into the trap of assuming sameness across families and cultures. A "theoretical myth of sameness," or "the conventional ideology ... that minority families are no different than non-minority families," is a common belief in the social sciences that must be challenged in order to provide competent and truly helpful assistance to a heterogeneous host of caregivers (Hardy, 1989, p. 18).

In their 20-year review of issues of race, culture, and ethnicity in the caregiving literature, Dilworth-Anderson and colleagues (2002) noted key differences among racially and ethnically diverse groups regarding social support (extended informal support networks), negative effects (burden, depression), coping (spirituality), and cultural effects (cultural rules and guidelines). Although a helpful starting point, noticing these differences in how caregiving is done and how it affects caregivers is not enough. Helping professionals must go beyond differences in outcome, seeking to understand differences in process and the meanings and intents that drive these processes. Important questions emerge that focus on both outcome (burden, depression) and process (values, meanings, intent) for caregivers: How do the concepts of responsibility and respect factor into your decision-making processes as a caregiver? What are your beliefs about the aging of a body and your role in caring for an ailing loved one? Though few, a number of exemplary studies are now focusing on these types of questions and are attempting to understand how differing cultural values and meanings drive process and outcome for caregivers (Dilworth-Anderson \& Gibson, 2002). 
Cloutterbuck and Mahoney (2003), studying African American caregivers, explored the cultural meaning of respect and how the application of this concept may help explain why African American caregivers seem to be able to carry a heavier caregiving load for longer periods of time with fewer reports of burden and depression than their white counterparts. Holroyd (2003) studied Chinese caregivers over a two-year period, focusing on how cultural and religious understandings of the body impact caregiving decisions. In her study, Holroyd learned that the caring for a loved one experiencing bodily illness and decay can have profound personal and social ramifications for caregivers, thereby complicating greatly the entire caregiving process. Cultural aspects of caregiving continue to be understudied among a variety of cultures, including Native Americans, for example.

Research on gender differences in caregiving has highlighted the fact that women provide the greatest bulk of caregiving service. "Women tend to provide more personal and instrumental care than men [and] are more likely to report impaired well-being than men because of their greater sensitivity to negative feelings, greater willingness to report negative feelings, and less effective coping styles" (Pinquart \& Sorensen, 2003b). Research has also shown that men are more likely to engage additional helpers and less likely to refer to caregiving as emotional work (Allen, 1994; Miller \& Kaufman, 1996). Because of the tremendous burden they carry and the multiple roles they attempt to fulfill on a daily basis, it is easy to understand why many female caregivers may struggle to balance tremendous family and employment responsibilities while at the same time they may also be experiencing poorer emotional health (Navaie-Waliser, Spriggs, \& Feldman, 2002).

Other studies suggest that many husbands adopt a task-oriented approach to caregiving, thereby maintaining an authority position, while wives work to preserve autonomy for their husbands and connection in the relationship (Corcoran, 1992; Wilken et al., 1996). Future research, which can better identify the meanings and intent of genderspecific behavior, would serve to clarify whether men and women do focus on or prefer instrumental versus affiliative support. Wilken and colleagues (1996) suggested that caregivers who feel adept at providing both types of care might be less vulnerable to strain. In the end, it is important to note that caregiving is carried out (objective) and experienced (subjective) differently by women and men and across diverse cultures.

\section{LEVELS OF INTERVENTION: FROM RESPITE CARE TO PSYCHOTHERAPY}

Over the years, helping professionals have worked with caregivers on a number of levels. Although the intent of most interventions is similar (alleviate burden, foster coping, build support), the approaches do differ in major ways (see Sorensen, Pinquart, \& Duberstein, 2002 for a comprehensive review of the effectiveness of caregiver interventions).

\section{Respite Care}

As the stressors of caregiving begin to mount, numerous caregivers have found help through the use of respite care (Zarit, Johansson, \& Jarrott, 1998). Respite care takes many forms, ranging from adult day care services to in-home care to friends and family helping out (see Zarit et al., 1998 for more detailed review). The primary purpose of this formal respite care is to ease the burden of the caregiver, and where possible, provide a safe and socially engaging experience for the recipient. Research has shown that caregiver satisfaction with respite care services is usually quite high, and even 
though respite care can trigger emotional (guilt over leaving a loved one in the care of others) and financial struggles, many caregivers would utilize these services more often if they could (Zarit et al., 1998). Respite care provides a needed break from caregiver responsibilities. Though limited by cost and availability, it appears to be a helpful coping technique (Gaugler et al., 2003; Sorensen et al., 2002). Unfortunately, research has yet to clearly link caregiver satisfaction with enduring positive change in caregiver wellbeing (Myers, 2003).

\section{Support Groups}

Support groups for caregivers are perhaps the most well-known and popular form of intervention. Having arisen out of a grass roots effort to support caregivers of persons with Alzheimer's disease, support groups are a key setting for providing important information, combating isolation, and fostering collaborative problem solving (Zarit \& Zarit, 1998). The scant research on support group outcomes is mixed and has not yet shown that groups are effective at reducing caregiver burden (Corcoran et al., 2000; Myers, 2003). Nevertheless, support groups remain popular, likely because they are widely available, affordable, and provide a close-knit support system of those who share a similar experience. Some researchers have cautioned that support groups may not always be monitored for accuracy of information and advice or run by leaders with training; therefore, support groups "should not be the first line of help or the only source of help for caregivers" (Zarit \& Zarit, 1998, p. 314; Jacobs, 1997).

\section{Psychoeducation/Skills Training}

Providing families with education and skills regarding the course of a loved one's illness and accompanying care recipient needs, specific caregiving tasks, available resources, and successful coping strategies can serve to greatly reduce avoidable struggle and conflict. Psychoeducational interventions differ from traditional support groups in that they are typically more formal and research based. Such programs are consistently led by a trained leader and can take many forms (all day seminar, multi-week group, lecture, distribution of written materials); whatever the format, psychoeducational interventions have proven effective in reducing caregiver burden and depression and care receiver symptoms (Sorensen et al., 2002). A simple but effective example of the potential positive impact of timely education is noted by Zarit \& Zarit (1998). In their work, they cite several touching examples of helping caregivers learn that a calming and reassuring response is more effective than a corrective and argumentative one when care recipients wish to engage in dangerous activities. Although there has been some disagreement over the long-term benefits, psychoeducational approaches to caregiver intervention consistently rate among the most effective type of interventions (Corcoran et al., 2000; Sorensen et al., 2002).

\section{Psychotherapy}

Psychotherapy as an intervention can be described as a therapeutic relationship between a caregiver and a trained mental health professional (Sorensen et al., 2002). As Sorensen and colleagues (2002) noted in their review, traditional cognitive-behavioral approaches to therapy with caregivers serve to help them "develop problem-solving abilities by focusing on time management, overload, and emotional reactivity management, and help the caregiver reengage in pleasant activities and positive experiences" (p. 358). Therapy may be short or long term, strength or problem focused; in the end, therapy provides caregivers with a set time to discuss concerns and receive individual attention from a trained professional poised to help in 
burden management and problem resolution (Zarit \& Zarit, 1998). The Zarits (1998) note that therapy may be especially helpful in situations where the caregiver environment is particularly problematic. In such circumstances, there is evidence that psychotherapy interventions with caregivers have resulted in gains related to caregiving burden and wellbeing (Sorensen et al., 2002).

\section{Common Factors}

\section{Among Interventions}

Whether the intervention involves a support group or a professional therapist, effective interventions seem to share a few common factors. Excluding respite care, all of the above-mentioned interventions rely heavily upon three treatment strategies highlighted in a model developed by Zarit and colleagues over years of practice and research (Zarit \& Zarit, 1998). In this model, the authors describe the need for interventions to provide information, foster problem solving, and provide support. Over time, this three-pronged approach to intervention has received consistent empirical support, regardless of the mode of intervention (Zarit \& Zarit, 1998).

\section{WHAT ABOUT THE FAMILY?}

In 1989, Steven Zarit wrote a provocative editorial titled, "Do we really need another 'stress and caregiving' study?" In his paper, Zarit argued that more attention should be placed on identifying effective (both in terms of cost and suffering) interventions than on re-establishing a well-known fact, namely that caregiving can be very stressful. Apparently researchers have heeded Zarit's call, evidenced by two major meta-analyses of caregiver interventions since his original piece was published (Knight, Lutzky, \& Macofsky-Urban, 1993; Sorensen et al., 2002). Although there has been a clearly marked increase in intervention studies, most continue to focus on and intervene at the individual level. Though the language of family caregiving or providing care in a family setting is often employed, few interventions actually attempt to address and resolve specific family concerns or foster change and healing within strained family relationships (Mitrani \& Czaja, 2000).

This oversight may prove costly because research on caregiving has consistently shown that the quality of the relationship between caregivers and care recipients, as well as between caregivers and other family members, directly affects caregiver outcomes (Lyons et al., 2002; Miller, 1990). In a review and commentary on caregiving literature, Miller (1990) noted numerous empirical studies have found that a significant amount of caregiver burden can be attributed to conflictual family relationships. For his support, Miller points to studies reporting that family relationship quality may predict a greater amount of perceived caregiver effectiveness than care recipient impairment (Townsend \& Noelker, 1987) and level of conflict between caregiver and care recipient may predict a significant amount of caregiver strain and negative affect (Sheehan \& Nuttall, 1988). A significant body of research has already demonstrated that conflict in noncaregiving couples has a negative effect on health variables ranging from immunology to daily health practices (Keicolt-Glaser \& Newton, 2001). Factors in family relationships seem to have a major impact on caregiver experience, yet it is ironic that two major reviews of the caregiving experience and intervention did not include family variables related to caregiver outcomes (see Pinquart \& Sorensen, 2003a; Sorensen et al., 2002).

\section{The Family Meeting}

An appealing approach to addressing family issues, particularly to fostering cooperation among family caregivers, is the family 
meeting (Zarit \& Zarit, 1998). The guiding principles behind the family meeting are rooted in family systems theory.

From a family systems perspective, it is [very] useful to get a sense of how a family functions, for example, who is influential and who is not, who is close to and distant from whom, and what the roles of the caregiver and care recipient have been in the family. (Zarit \& Zarit, 1998, p. 310)

Guided by a trained clinician, the family meeting provides an appropriate venue to ensure all family members have the same accurate information about the loved one's illness and that each understands the level of care required. Once this occurs, the meeting can focus on what the primary caregiver wants and needs and the role of other family members in the caregiving process. The clinician can help the family form a plan for future action by being "neutral and supportive" and guiding them towards a resolution of conflict; however, "the therapist should ... not try to change family process or treat long-standing problems or to redress the balance of power within the family" (Zarit \& Zarit, 1998, p. 312). This is the role of family therapy.

\section{Other Family-Based \\ Interventions for Caregiving Families: Empirical Support}

There are a few studies that tested the effectiveness of a structured, family-based intervention for caregiving families. Two noteworthy studies provide excellent examples of how family caregiving systems can be engaged and helped through family-based interventions. In their randomized controlled trial, Mittelman, Ferris, Shulman, Steinberg, and Levin (1996) provided four family counseling sessions as a part of their broader protocol. In those sessions, the counselor helped families address and resolve problems regarding patient behavior, improve communication among family members, and increase emotional and instrumental support for the primary caregiver. Families who participated in the broad-based intervention were able to care for their loved one at home for longer periods of time than those in the control group, especially throughout beginning stages of dementia when placement in a care facility may not be necessary.

A second study, reported by Mitrani and Czaja (2000), demonstrates how culturally sensitive home-based family therapy may be a particularly appropriate and effective intervention. Mitrani, Czaja, and colleagues applied Structural Ecosystemic Therapy (SET), a proven family systems-based therapy, to their work with Cuban caregiving families. In their sessions, members of the SET team worked in the home with caregiving families to build a healthy and functional family structure (hierarchy, alliance, communication), foster resonance (address caregiver and care recipient enmeshment), and resolve conflict. The SET intervention was one of nine active interventions tested in the REACH multisite initiative with caregivers and the only approach that evaluated a family systems-based intervention. At six month follow-up, the SET approach had not only established itself as effective when compared to inactive groups; it was also one of two interventions that had significantly reduced depressive symptoms in caregivers (Gitlin et al., 2003).

\section{A THEORETICAL FRAMEWORK FOR EXTENDING FAMILY-BASED WORK}

In a recent paper, Pillemer and colleagues (2003) argued that although intertwined, good theory precedes quality intervention design and testing. Because of the oversight regarding and need for increased research into 
family-based interventions with caregivers, it is important that a solid, family-based theoretical framework is laid upon which to build an intervention framework. Perhaps a combination of developmental and relational ethics theories could serve as an initial launching point for a richer discussion of familybased theory and its implications for helping professionals on the front lines with caregiving families.

\section{Developmental Theory}

It is important to recognize a few key developmental factors that may result in strain for many caregivers and recipients. The eighth stage of Erik Erickson's (1963) theory of psychosocial development provides a useful lens through which to view the one major struggle of later-life families. According to the theory, as older adults approach death, they naturally grapple to find the meaning in their existence: Has my life mattered? Have I accomplished my goals? Have I made a difference? These are questions that may arise as older adults struggle with ego integrity versus despair (Crain, 1992; Hargrave \& Anderson, 1997). This normative process of sifting through past experiences as motivated by the nearness of death has been termed life review (Butler, 1963).

Likewise, many adult children and spouses are faced with a similar process as they are drawn towards their ailing loved one because of the perceived finality of death (Carstensen, 1998). In many cases, this review of the relational landscape may not immediately yield feelings of contentment and appreciation; in fact, it may only amplify family members' struggles with past contention and inflicted hurts. The pressure to "make things right" in a short period of time can greatly complicate family processes. This stage of life and the naturally occurring process of life review places older adults and their families in a vulnerable position as they recall intense and painful interactions, wounds, and conflicts from their past (Hargrave \& Anderson, 1997). It is at this stage and in this context that the bulk of caregiving often occurs. How can family members balance the opposing forces of providing care out of a sense of love and duty and a looming sense of frustration and anger over past hurts?

\section{Relational Ethics}

Boszormenyi-Nagy's theory of relational ethics, commonly known as contextual theory, provides potential answers to this difficult question and a useful lens for clinicians dealing with caregiving issues (Boszormenyi-Nagy \& Krasner, 1986; see Hargrave \& Anderson, 1992 for a beautiful and clear application of contextual theory to common clinical problems of aging families). Relational ethics are concerned "with the subjective balance of trustworthiness, justice, loyalty, merit, and entitlement among members of a relationship" (Hargrave \& Anderson, 1997, p. 63). When family members can interact in a balanced or fair manner, where both can give and take without manipulation, trustworthiness is developed. According to this theory, trustworthiness is the key to family loyalty and strength across generations (Hargrave \& Anderson, 1997). Conversely, when trust erodes, family dysfunction often follows (Anderson \& Hargrave, 1990).

Symptoms of intergenerational conflict or dysfunction can be seen as a sign of an unbalanced relational account. These accounts or ledgers can be described as ongoing legacies of emotional commitments and obligations (Everett, Russell, \& Keller, 1992; Spark, 1974). Simply put, multigenerational ledgers are accounts of relational charges (hurts) and credits (benefits) accrued by family members over time (Boszormenyi-Nagy, 1974). 
This metaphor of families making transactions (interacting) with overdrawn accounts (unbalanced ledgers) can be readily applied to caregiving families. Imagine an adult daughter who is providing care for her ailing father. As is typical of many caregivers, she is trying to balance the demands of her own family and a job while looking after her father. Although her cultural and personal expectations are that she provides loving and dutiful care, she is often resentful and angry. As a result, she is experiencing increased conflict in her own home and feels criticized by her own siblings regarding the type of care she is providing for their father.

In a family meeting or family therapy session, would it not be common for some of the following questions to be raised? "Why was I the one selected to be the primary caregiver? I have always done more work than my brothers and sisters." "This is just like it was when we were kids; she was always in control. How are we supposed to help when she never lets us get involved?" "How can I feel loving and connected to Dad when he was never part of my life? Even when he was home from work, he was always so angry and violent. Am I supposed to forget all of that pain?" Though often ignored in the research literature, clinicians recognize that expectations regarding loyalty and fairness can drive caregiving decisions and experience (Hargrave \& Anderson, 1997; Jacobs, 1997; Sandberg, 1999).

Therefore, a major task for many caregiving families is to reconcile themselves with painful issues from their past. As painful as the process may be, contextual theory claims that revisiting these encounters can prepare the way for a balancing of intergenerational accounts. Therefore, "older [adults and their] families need to do the work of communicating about, reconciling with, and forgiving the past. In these actions, the ground work for trustworthy relations[hips] is constructed" (Hargrave \& Anderson, 1997, p. 65).

\section{PRACTICAL INTERVENTIONS FOR FAMILY-FOCUSED WORKERS}

There are times when family therapy is needed to address long-standing problems and address issues of power and control in caregiving families (Zarit \& Zarit, 1998). Developmental and contextual theories provide a base from which the following interventions are built.

\section{The Life Review}

Life review interventions (Butler, 1963) are based upon the concept that older adults and their families will naturally undergo a period of reflection about the purpose and meaning of their existence as death approaches. Because issues of fairness, loyalty, and entitlement become evident as past experiences are recalled through the life review, important details regarding the family's intergenerational ledger can be gleaned in this process. With this increased awareness of how and when injustices first occurred, aging families are better able to understand their own relationship with both the past (their own parents) and the future (their children). This process allows family members to identify with each other in healthier ways through appreciation and understanding. It is only through these new, fairer interactions that trust is rebuilt and ledgers are balanced. The life review exercise, in the presence of family, is one action strategy to foster this healing (Spark, 1974).

In addition, the life review can be used as a simple yet powerful tool for connecting and calming when relationships have become strained in more acute, rather than chronic, ways. Hargrave (2002) described a clinical case in which an adult daughter Robin (age 60) and her mother Maggie (84) came to family therapy. Shortly after Maggie had left her long-term residence and moved to Robin's home, the relationship environment 
began to sour. Although Maggie was still able to function independently in a number of areas at these beginning stages of Alzheimer's disease, she felt bossed around by her daughter; Robin was frustrated over her mother's increasing dependence and angry outbursts. Perhaps in an attempt to connect and calm, Dr. Hargrave engaged Robin and Maggie in a conversation about the "good old days."

This simple looking back led Robin and Maggie into a conversation about a "good husband" and a "good father" and how they both missed him. At one point, Maggie slid her hand into her daughter's, saying, "Well, I guess you and I are what we have now." Robin came to realize that the pressures of caregiving had caused her to press too hard to get things done in a hurry. In turn, this likely frustrated her mother at a level that she could not verbalize, and so she wound up expressing herself [by throwing] a hairbrush. Robin was eager to make changes. She sought ways to connect with her mother, like sharing old pictures. Robin learned how to keep the storytelling from becoming repetitive, and picked out two personal events each week that helped bring back old times. Maggie had no more violent outbursts... [and during] those months, Robin had learned to connect with her mom emotionally... [leading her to call this period] some of the most tender times in our lives. (Hargrave, 2002, p. 26)

\section{The Work of Forgiveness}

A second approach to balancing intergenerational accounts in later life is through the work of forgiveness. Interventions aimed at forgiveness may be particularly helpful in addressing "basic questions of love and trust in the family and rebuild[ing] relationships after severe family violations have occurred" (Hargrave \& Anderson, 1997, p. 68). For some individuals, increased awareness regarding their aging parent's own suffering, although extremely beneficial, will not be enough to compensate for long-standing injustices. For such families, the work of forgiveness can provide a more structured format for resolving long-standing relational indebtedness. The ability to resolve these longstanding conflicts may be particularly valuable when the person experiencing the hurt is valiantly trying to provide care for the one who did the hurting.

Hargrave and Anderson (1997) described forgiveness as two distinct processes, exoneration and forgiveness. "Exoneration means the victim is able to lift the load of culpability from the victimizer while making significant connection and identification, whereas forgiveness means that the victim and victimizer are actually able to restore a loving and trustworthy relationship" (p. 68). Although it is not possible or wise for all individuals to achieve this definition of forgiveness (that is, in cases where a victimizer continues to make unjust demands on the victim's account), it is possible for most individuals to exonerate their ailing loved one.

The work of exoneration can be broken down into two stations (Hargrave \& Anderson, 1992; Hargrave, 1994). The first station, insight, involves gaining a cognitive awareness of the experience of another and how past pains have been transferred to current relationships (Hargrave \& Anderson, 1997). Not until the hurt party can identify relationship patterns that perpetuate pain can he or she stop the process. The second station, understanding, seeks to foster a level of identification with or even empathy for the victimizer's own suffering and efforts to change problematic patterns in his or her own life (Hargrave \& Anderson, 1997). Both the insight and the deeper level of understanding can be facilitated in a life review intervention.

The actual work of forgiveness also involves two stations. Giving the opportunity for compensation is a process whereby the 
victim allows the victimizer to change and rebuild the relationship by demonstrating trust and love over time in an effort to erase past injustices (Hargrave \& Anderson, 1997). This process allows the hurt party to test, in stages, the trustworthiness of the one who has previously inflicted pain. Only as trustworthiness is built can the ledger be rebalanced. The fourth station, the overt act of forgiveness, is where victim and victimizer work directly and honestly to confront and heal from past hurt through the "agreement, acknowledgement, and apology" (Hargrave \& Anderson, 1997, p. 4). During this last phase, family-focused workers must help the victim and victimizer come to a ball park agreement of what the hurt is, acknowledge that the wrongdoer is responsible for the wrongs committed, and facilitate an apology that demonstrates regret and a sincere desire to do better (Hargrave \& Anderson, 1997). This process, though extremely intense and only possible with two willing parties, can provide tremendous healing.

Returning to the previous hypothetical example of a family session with an adult daughter caregiver, her siblings, and an ailing father, how could a family therapist help facilitate exoneration or forgiveness and what difference would it make? A family therapist based in Contextual theory may seek to help children understand the experiences in their own father's life that might have led him to be more absent or violent than they or he wanted (insight). The family therapist might also help the children understand how the tendency to avoid or blow up surfaces at times in their own lives (understanding). The family therapist would also work to help the father, if cognitively able, to engage in a healing dialogue with his children in which he acknowledges past mistakes and takes responsibility for them (initial steps of forgiveness). Such difficult and healing dialogues between father and children and among siblings have a tremendous potential to increase support and unity among family members and reduce subjective burden, two factors that contribute greatly to the overall experience of a caregiver.

\section{FUTURE DIRECTIONS}

To paraphrase Zarit (1989), do we really need more studies that focus on the individual experience of caregivers and treatments that intervene on an individual level? If families are truly the primary and most basic support structure for providing care for ailing loved ones, future researchers and clinicians will need to combine together to successfully address three key areas relating to family caregiving (Zarit \& Zarit, 1998).

\section{Absence of Empirical Research Regarding Family-Based Interventions for Caregivers}

Though theoretically and logically sound, few studies have actually tested family-based models for caregiving families. Because of elevated divorce rates, greater distance between family members, and decreased health care coverage trends, more and more family members will be forced to face difficult issues as they struggle to provide care in a family setting. As a result, more and more practitioners will be called upon to help these families. Unfortunately, the amount of clinical research, even theoretical research, in this area is insufficient to support the looming need.

\section{Absence of Culturally Sensitive and Effective Family Interventions with Caregivers}

With exception of the work by Mitrani and Czaja (2000), very little is known about how to best intervene with families from a variety of cultural backgrounds. It is interesting to 
note that in a major meta-analysis of the caregiving intervention literature, race and culture is not addressed as a variable of interest (Sorensen et al., 2002). Do not racial and cultural differences impact the experience of caregiving? Should not interventions be tailored to meet the specific needs and highlight the unique strengths of specific groups? The continued under-appreciation of differences (Hardy, 1989) will prove costly in light of tremendous increases in diverse groups of the elderly over the next 50 years.

\section{Linking Research, Policy and Practice}

If researchers and clinicians are able to work collaboratively to identify and highlight culturally competent, effective familybased interventions for caregivers, there still remains the tremendous task of making those interventions available to those who need them most. For many families, treatment for any struggle related to relational, emotional, or mental health is simply out of their reach. Though demographics demand more emphasis and money be put into aging issues, such a change has yet to occur at a level that will be required. A significant shift is needed to make therapy for caregiving families readily available and truly beneficial.

\section{CONCLUSION}

In a heart-rending article, Jacobs (1997) pays homage to caregivers he works with, describing them as:

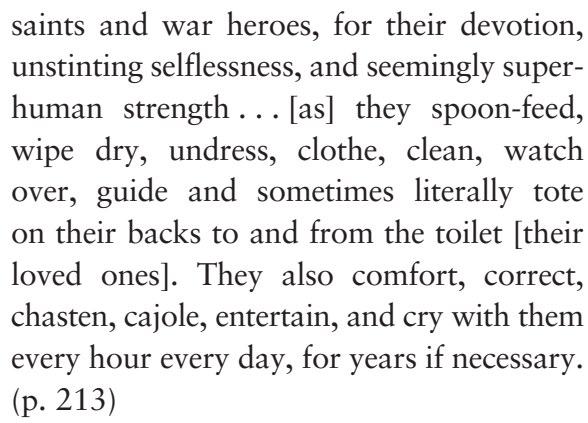

At times it is easy to forget that behind each of the thousands of caregiving studies is a family of real people trying to do the best they can. May we, as researchers and clinicians, keep that in mind as we push forward to help decrease burdens and increase uplifts for these noble families.

\section{REFERENCES}

Administration on Aging (AoA), U.S. Department of Health and Human Services. (2000). A profile of older Americans: 2000. Washington, DC: U.S. Government Printing Office.

Allen, S. M. (1994). Gender differences in spousal caregiving and unmet need for care. Journal of Gerontology, 49(4), S187-S195.

Anderson, W. T., \& Hargrave, T. D. (1990). Contextual family therapy and older people: Building trust in the intergenerational community. Journal of Family Therapy, 12, 311-320.

Armas, G. C. (2003). Worldwide population aging. In H. Cox (Ed.), Annual editions: Aging (pp. 3-4). Guilford, CT: McGraw-Hill.

Boszormenyi-Nagy, I. (1974). Ethical and practical implications of intergenerational family therapy: What is psychotherapy? Psychosomatic, 24, 261-268.

Boszormenyi-Nagy, I., \& Krasner, B. (1986). Between give and take: A clinical guide to contextual therapy. New York: Harper \& Row. 
Butler, R. N. (1963). The life review: An interpretation of reminiscence in the aged. Psychiatry, 26, 65-76.

Carstensen, L. (1998). Emotional functioning in old age. Paper presented at Gerontological Society of America Annual Conference, Philadelphia, PA.

Cloutterbuck, J., \& Mahoney, D. F. (2003). African American dementia caregivers. Dementia, 2(2), 221-243.

Conger, C. O., \& Marshall, E. S. (1998). Recreating life: Toward a theory of relationship development in acute home care. Qualitative Health Research, 8(4), 526-546.

Corcoran, M. A. (1992). Gender differences in dementia management plans of spousal caregivers: Implications for occupational therapy. American Journal of Occupational Therapy, 46(11), 1006-1012.

Corcoran, M. A., Fairchild-Kienlen, S., \& Phillips, J. H. (2000). Family treatment with caregivers of the elderly. In J. Corcoran (Ed.), Evidence-based social work practice with families (pp. 505-556). New York: Springer.

Crain, W. (1992). Theories of development: Concepts and applications. Englewood Cliffs, NJ: Prentice-Hall.

Dilworth-Anderson, P., \& Gibson, B. E. (2002). The cultural influence of values, norms, meanings, and perceptions in understanding dementia in ethnic minorities. Alzheimer Disease and Associated Disorders, 16(Supp. 2), S56-S63.

Dilworth-Anderson, P., Williams, I. C., \& Gibson, B. E. (2002). Issues of race, ethnicity, and culture in caregiving research: A 20-year review (1980-2000). The Gerontologist, 42(2), 237-272.

Erikson, E. H. (1963). Childhood and society (2nd ed.). New York: Norton.

Everett, C. A., Russell, C. S., \& Keller, J. (1992). Family therapy glossary (pp. 1-40). Washington, DC: The American Association for Marriage and Family Therapy.

Gaugler, J. E., Jarrott, S. E., Zarit, S. H., Stephens, M. P., Townsend, A., \& Greene, R. (2003). Adult day service use and reductions in caregiving hours: Effects on stress and psychological well-being for dementia caregivers. International Journal of Geriatric Psychiatry, 18, 55-62.

Gitlin, L. N., Belle, S. H., Burgio, L. D., Czaja, S. J., Mahoney, D., GallagherThompson, D., et al. (2003). Effect of multicomponent interventions on caregiver burden and depression: The REACH multisite initiative at 6-month follow-up. Psychology and Aging, 18(3), 361-374.

Hardy, K. V. (1989). The theoretical myth of sameness: A critical issue in family therapy training and treatment. Journal of Psychotherapy and the Family, $6(1-2), 17-33$.

Hargrave, T. (2002). My child, my mother. Modern Maturity, 45(2), 25-26.

Hargrave, T. D., \& Anderson, W. T. (1992). Finishing well: Aging and reparation in the intergenerational family. New York: Brunner/Mazel.

Hargrave, T. D., \& Anderson, W. T. (1997). Finishing well: A contextual family therapy approach to the aging family. In T.D. Hargrave \& S. M. Hanna (Eds.), The aging family (pp. 61-80). New York: Brunner/Mazel.

Hargrave, T. D., \& Hanna, S. M. (1997). The aging family: New visions in theory, practice, and reality. New York: Brunner/Mazel.

Holroyd, E. (2003). Hong Kong Chinese family caregiving: Cultural categories of bodily order and location of self. Qualitative Health Research, 13(2), 158-170.

Jacobs, B. J. (1997). In sickness and health: At the caregiver support group. Families, Systems and Health, 15(2), 213-220. 
Kiecolt-Glaser, J. K., \& Newton, T. L. (2001). Marriage and health: His and hers. Psychological Bulletin, 127(4), 472-503.

Knight, B. G., Lutzky, S. M., \& Macofsky-Urban, F. (1993). A meta-analytic review of interventions for caregiver distress: Recommendations for future research. The Gerontologist, 33, 240-248.

Kramer, B. J. (1997). Gain in the caregiving experience: Where are we? What next? The Gerontologist, 17(2), 218-232.

Lyons, K. S., Zarit, S. H., Sayer, A. G., \& Whitlatch, C. J. (2002). Caregiving as a dyadic process: Perspectives from caregiver and receiver. Journal of Gerontology: Psychological Sciences, 57B(3), P195-P204.

Miller, B., \& Kaufman, J. E. (1996). Beyond gender stereotypes: Spouse caregivers of persons with dementia. Journal of Aging Studies, 10(3), 189-204.

Miller, R. B. (1990). Building bridges between family policy, research, and practice. Paper presented at Seventh National Forum on Aging, Lincoln, NE.

Mitrani, V. B., \& Czaja, S. J. (2000). Family-based therapy for dementia caregivers: Clinical observations. Aging and Mental Health, 4(3), 200-209.

Mittelman, M. S., Ferris, S. H., Shulman, E., Steinberg, G., \& Levin, B. (1996). A family intervention to delay nursing home placement of patients with Alzheimer disease. Journal of the American Medical Association, 276(21), 1725-1731.

Myers, J. E. (2003). Coping with caregiving stress: A wellness-oriented, strengthsbased, approach for family counselors. The Family Journal: Counseling and Therapy for Couples and Families, 11(2), 153-161.

Navaie-Waliser, M., Spriggs, A., \& Feldman, P. H. (2002). Informal caregiving: Differential experiences by gender. Medical Care, 40(12), 1249-1259.

Pillemer, K., Suitor, J. J., \& Wethington, E. (2003). Integrating theory, basic research, and intervention: Two case studies from caregiving research. The Gerontologist, 43(SI1), 19-28.

Pinquart, M., \& Sorensen, S. (2003a). Associations of stressors and uplifts of caregiving with caregiver burden and depressive mood: A meta-analysis. Journal of Gerontology: Psychological Sciences, 58B(2), 112-128.

Pinquart, M., \& Sorensen, S. (2003b). Differences between caregivers and noncaregivers in psychological health and physical health: A meta-analysis. Psychology and Aging, 18(2), 250-267.

Sandberg, J. G. (1999). "It just isn't fair": Helping older families balance their ledgers before the note comes due. Family Relations, 48, 177-179.

Sandberg, J. G., \& Platt, J. J. (2001). Family therapy and issues of aging. In M. M. MacFarlane (Ed.), Family therapy and mental health: Innovations in theory and practice (pp. 361-388). Binghamton, NY: The Haworth Clinical Practice Press.

Sheehan, N., \& Nuttall, P. (1988). Conflict, emotion, and personal strain among family caregivers. Family Relations, 37, 92-98.

Sorensen, S., Pinquart, M., \& Duberstein, P. (2002). How effective are interventions with caregivers? An updated meta-analysis. The Gerontologist, 42(3), 356-372.

Spark, G. M. (1974). Grandparents and intergenerational family therapy. Family Process, 13(2), 225-237.

Townsend, A., \& Noelker, L. (1987). The impact of family relationships on perceived caregiving effectiveness. In T. Brubaker (Ed.), Aging, health, and family: Long term care (pp. 80-99). Newbury Park, CA: Sage.

Wilken, C. S., Altergott, K., \& Sandberg, J. (1996). Spouses' self-perceptions as caregivers: The influence of feminine and masculine sex-role orientation on 
caring for confused and non-confused partners. American Journal of Alzheimer's Disease, 11(6), 37-42.

Zarit, S. H. (1989). Do we need another "stress and caregiving" study? The Gerontologist, 29, 481-483.

Zarit, S. H., Johansson, L., \& Jarrott, S. E. (1998). Family caregiving: Stresses, social programs, and clinical interventions. In I. H. Nadhus, G. R. Vanden-Bos, S. Bera, \& P. Fromholt (Eds.), Clinical Geropsychology (pp. 345-360). Washington, DC: American Psychological Association.

Zarit, S. H., \& Zarit, J. M. (1998). Mental disorders in older adults: Fundamentals of assessment and treatment. New York: Guilford. 